\title{
Evolutions et régulations des illégalismes populaires en France depuis le début des années 1980
}

\section{Laurent Bonelli}

\section{(2) OpenEdition Journals}

Édition électronique

URL : http://journals.openedition.org/conflits/957

DOI : $10.4000 /$ conflits.957

ISSN : $1777-5345$

Éditeur :

CCLS - Centre d'études sur les conflits lilberté et sécurité, L'Harmattan

Édition imprimée

Date de publication : 1 septembre 2003

Pagination : 9-42

ISBN : 2-7475-5377-9

ISSN : 1157-996X

Référence électronique

Laurent Bonelli, «Evolutions et régulations des illégalismes populaires en France depuis le début des années 1980 », Cultures \& Conflits [En ligne], 51 | automne 2003, mis en ligne le 02 février 2004, consulté le 30 mars 2021. URL : http://journals.openedition.org/conflits/957 ; DOI : https://doi.org/ $10.4000 /$ conflits.957

Ce document a été généré automatiquement le 30 mars 2021.

Creative Commons License 


\title{
Evolutions et régulations des illégalismes populaires en France depuis le début des années 1980
}

\author{
Laurent Bonelli
}

«Et la répression, aussi cruelle que la récession »

La Rumeur, L'ombre dans la mesure, EMI music France 2002

«L'explosion» de l'insécurité dans les banlieues françaises est devenue un sujet incontournable du débat politique, électoral et médiatique. Les discours inquiets ou alarmistes, les dossiers spéciaux et les reportages spectaculaires se succèdent et se multiplient reléguant au second plan des pans entiers de l'actualité sociale et politique du pays. Analystes, «experts » et essayistes de la sécurité prophétisent sur fond de cartes exponentielles de la délinquance, l'avènement de zones de "non-droit " aux mains de délinquants toujours plus jeunes, plus récidivistes et plus violents, alors que les différents partis politiques, toutes tendances confondues, invoquent la « demande de sécurité » de leurs électeurs pour réclamer une action plus énergique de la police et de la justice. Depuis le milieu des années 1990, la sécurité urbaine est de la sorte devenue l'une des principales priorités des différents gouvernements, qui y consacrent des moyens importants, matériels et législatifs.

Pour comprendre cette inflation extraordinaire du thème de la sécurité dans notre société, il importe d'analyser la configuration -comme l'entend Norbert Elias ${ }^{1}$ - dans laquelle elle prend sens, c'est-à-dire de restituer l'ensemble des chaînes d'interdépendance qui relient entre eux de multiples agents sociaux appartenant à des univers aussi différents que la police, la politique, la presse, l'université, l'entreprise, etc. Ceci implique dès lors de mettre à jour les équilibres fluctuants des tensions et des relations de pouvoir entre ces différents protagonistes, en insistant sur leur historicité et les visions du monde qu'elles véhiculent. En effet, un « problème social » n'existe pas en lui-même: il suppose pour accéder à ce statut un véritable travail social. Toute transformation sociale ne devient pas un problème social et rendre visible une situation particulière «suppose l'action de groupes socialement intéressés à produire une 
nouvelle catégorie de perception du monde social afin d'agir sur ce dernier $»^{2}$. Ce qui revient à dire qu'aux changements objectifs, sans lesquels le problème ne se poserait pas, s'ajoute un travail spécifique d'énonciation et de formulations publiques, c'est-à-dire une entreprise de mobilisation qu'il faut à chaque fois questionner et mettre à jour. $C^{\prime}$ 'est ce que je me propose de faire ici, en reliant les transformations morphologiques des quartiers populaires français, aux évolutions des modes d'appréhension de la "violence » des adolescents de ces quartiers et les effets de l'engagement sans cesse accru des forces de police dans la résolution de ces « désordres urbains ».

\section{Dégradation sociale et désaffiliation}

L'expression "quartiers populaires" fait essentiellement référence aux grands ensembles des périphéries urbaines appelés en France «banlieues» et, dans une moindre mesure, aux quartiers anciennement ouvriers que l'on retrouve au centre des grandes villes.

Ces quartiers ont connu entre les années 1960 et aujourd'hui de grandes évolutions. Construits entre les années 1950 et les années 1970, ces grands ensembles aux noms évocateurs de « cité des 4000 » (pour 4000 logements) à la Courneuve, « cité des 3000 » à Aulnay sous bois, etc. visaient à apporter une réponse rationnelle et planifiée à la question du logement et plus largement au développement urbain. Ils étaient destinés à résorber les taudis, nombreux à cette époque, à améliorer la condition des familles « modestes » et à rapprocher les travailleurs de leurs usines ${ }^{3}$. Ces efforts pour « loger le peuple » sont en effet à mettre en parallèle avec l'augmentation des effectifs ouvriers qui passent de 6.485 .000 en 1954 (33,2\% de la population active) à 8.191.000 en 1975 $(37,2 \%)$ avec une croissance de $1 \%$ par an entre 1954 et 1978. En 1974, la concentration de la main d'œuvre dans les grandes usines a atteint son zénith, avec 2.600.000 de postes d'ouvriers non qualifiés de type industriel ${ }^{4}$. Si la vision rétrospective de cette période tend à la faire apparaître comme un âge d'or qu'elle ne fut sans doute pas ${ }^{5}$, elle n'en représente pas moins une période de progrès social pour nombre de familles ouvrières françaises qui pouvaient enfin accéder au confort (eau courante, électricité, etc.) et pour qui l'avenir semblait enfin ouvert.

Le départ progressif des ménages les plus aisés qui accèdent à la propriété, l'arrivée de familles immigrées et la précarisation de ceux qui restent sous l'effet de la crise économique vont profondément modifier la morphologie sociale de ces quartiers.

D'abord, sous l'effet des politiques libérales en matière de logement promues durant les années 1970 (aide à la pierre), les couches les plus favorisées de ces quartiers (principalement les ouvriers qualifiés [OQ]) les ont progressivement désertés pour faire construire des pavillons dans les multiples lotissements qui fleurissent en France à cette époque ${ }^{6}$. Ce mouvement, qui s'inscrit dans la continuité de leur ascension sociale et résidentielle, est à la fois une cause et une conséquence de l'évolution des cités HLM. D'un côté, ce dépeuplement est en partie motivé par l'arrivée de familles immigrées (algériennes et marocaines surtout), relogées par les préfectures ${ }^{7}$. En effet, malgré leurs conditions précaires d'habitat, ces populations étaient, dans les années 1970-80, largement tenues à l'écart du parc social. Ce n'est qu'au prix d'une politique préfectorale volontariste qu'elles purent y accéder, accélérant le départ des $O Q$ et des classes moyennes : les bâtiments qui se vident sont d'abord ceux qui ont servi à leur relogement. Dans l'autre sens, les conséquences - économiques notamment - de la vacance des immeubles qu'entraînent ces départs vont amener les bailleurs sociaux (privés ou municipaux) à ouvrir le parc HLM à des familles qu'ils refusaient jusque-là. 
Ils vont de la sorte transformer la composition sociale et communautaire de ces quartiers, précipitant par là même les départs et renforçant la concentration ${ }^{8}$.

A la même époque, la crise économique a durement frappé les emplois industriels (particulièrement non qualifiés) qui occupaient la majorité des habitants de ces zones : les entreprises ont fait d'importants efforts d'automatisation et les fabrications consommatrices de main-d'œuvre peu qualifiée ont été supplantées par les importations en provenance de pays du sud. Entre 1975 et 1999, le nombre d'ouvriers non qualifiés passe au niveau national de 3.840 .000 à 2.163 .000 , soit une diminution de $44 \%$. Les emplois non qualifiés qui disparaissent sont concentrés dans la production industrielle. Dans les secteurs du textile, de la confection, du travail du bois, et du travail du cuir, les trois quarts des emplois d'ouvriers non qualifiés ont ainsi disparu en vingt ans ${ }^{9}$. Cette situation concerne particulièrement les immigrés. En 1999, on comptait 2.100 .000 actifs immigrés (8,1\% de l'ensemble des actifs). Alors que les ouvriers représentent $26,3 \%$ de la population active, ce taux est de $44,1 \%$ chez les immigrés. Il monte même à $58,2 \%$ pour les Marocains et à $48,7 \%$ pour les Algériens. Les proportions sont plus élevées encore au niveau de l'emploi ouvrier non qualifié $(9,2 \%$ de la population active), qui occupe $19,1 \%$ des immigrés ; $31 \%$ des Marocains et $21,5 \%$ des Algériens ${ }^{10}$.

Les transformations induites par le passage à un modèle post-fordiste de production vont très largement déstructurer le monde ouvrier traditionnel. Le chômage de masse et la précarisation de l'emploi non qualifié vont en effet y réintroduire une insécurité et une imprévisibilité que l'avènement d'une société salariale (basée sur la croissance économique et un état social fort) avait largement réduite. Cette désobjectivation du salariat va simultanément déstabiliser les stables et créer de la désaffiliation.

Déjà avant 1975, le chômage des ouvriers était supérieur à celui des autres catégories sociales. Les ouvriers subissaient, plus directement que les autres salariés, les conséquences des transformations de l'appareil de production. Avec la crise, le chômage a fortement augmenté et notamment celui des travailleurs les plus âgés. Les employés et les ouvriers sont les catégories socioprofessionnelles les plus touchées. En 1999, les taux de chômage de ces catégories sont supérieurs à $14 \%$, et pour les immigrés, ils sont supérieurs à $21 \%$.

Ensuite, les transformations industrielles et notamment la production à flux tendu va généraliser le recours aux intérimaires et aux emplois temporaires. En 1995, 14\% des ouvriers, $25 \%$ des ouvriers non qualifiés ont un contrat à durée limitée : intérim, apprentissage, contrat à durée déterminée ou stage en entreprise. La proportion de tels emplois n'est que de $9 \%$ parmi l'ensemble des salariés ${ }^{11}$. En mars $2001,17 \%$ des salariés non qualifiés sont en CDD, en intérim, ou en stage contre $7 \%$ des salariés plus qualifiés. En 1982, les formes particulières d'emploi étaient essentiellement des CDD et ne concernaient que $4 \%$ des emplois non qualifiés. Si les CDD et les stages se sont développés de façon assez uniforme dans toutes les professions non qualifiées, les contrats d'intérim sont spécifiques du monde ouvrier. En mars 2001, le taux d'intérim dépasse souvent les $10 \%$ au sein des professions d'ouvriers non qualifiés ${ }^{12}$. On assiste ainsi simultanément dans ces quartiers à une baisse du niveau d'activité (nombre de gens occupant un emploi) et à une précarisation générale des statuts.

Ces phénomènes, qui modifient radicalement la structure sociale des classes populaires, ont également des conséquences symboliques: c'est toute l'économie politique des signes et des schèmes cognitifs qui structuraient leur rapport au monde qui se 
transforme. On ne peut de la sorte pas comprendre les évolutions de ces milieux sans envisager dans le même temps ces deux dimensions. Ceci est particulièrement nécessaire lorsque l'on veut penser la question des « déviances » juvéniles. D'un côté, celles-ci se redéfinissent sous l'effet des transformations de l'accès à l'emploi non qualifié, des modes de reproduction et de contrôle antérieurs; de l'autre, c'est la toile de significations dans laquelle elles étaient prises (et notamment l'idée « qu'il faut que jeunesse se passe ») qui se délite.

La «violence» de fractions des jeunes des classes populaires ne constitue pas un phénomène nouveau : il faut se rappeler des agressions perpétrées par les groupes de blousons noirs des années 1960, ou les loubards des années $1970^{13}$. Pour autant, ses modes de régulation, de même que sa perception se modifient largement. En effet, l'errance dans l'espace public propre à ces adolescents, qui se traduisait par une série de comportements "déviants" (violences verbales et physiques, petits vols, dégradations, etc.) prenait le plus souvent fin par l'intégration dans les fractions les plus déqualifiées du prolétariat industriel. Et loin de s'opposer à la culture de l'atelier, les valeurs dont ils étaient porteurs (virilité, violence, anti-autoritarisme, etc.) y trouvaient un réceptacle favorable. Il suffit de penser à la virilité des ateliers ${ }^{14}$, à la lutte contre le "petit chef", le contremaître. Ces valeurs nourrissaient même parfois l'action syndicale et politique. Au fil des années l'intégration professionnelle s'accompagnait du passage à un mode de vie plus conforme ("se ranger»), sans que s'opère réellement de rupture normative.

Aujourd'hui ces mêmes jeunes ne peuvent plus s'insérer dans un monde qui a largement décliné ${ }^{15}$, pas plus qu'ils ne peuvent occuper les nouveaux emplois sousqualifiés auxquels leur absence de qualification les voue objectivement. En effet, ceuxci se développent exclusivement dans les services ${ }^{16}$. Et s'il est exact de dire que le travail d'un caissier de supermarché est un travail à la chaîne, il diffère profondément de ce dernier par l'introduction du client, qui impose des formes de civilité et de comportements "normalisés » (docilité, politesse, voire déférence ${ }^{17}$ ) qui s'opposent aux valeurs de la rue. La différence sexuelle sur ce marché est d'ailleurs particulièrement manifeste. Les filles, socialisées différemment et poussées par le contrôle social familial élargi à quitter le plus rapidement la sphère domestique ${ }^{18}$ adoptent beaucoup plus volontiers ces manières d'être, qui leur permettent de mieux réussir scolairement et professionnellement que leurs homologues masculins. Dans ce contexte de concurrence entre les groupes pour l'occupation de ces emplois non qualifiés, les jeunes garçons porteurs de valeurs qui fonctionnent comme un véritable stigmate social sont particulièrement désavantagés.

Dans le même temps, la massification de l'enseignement en France a prolongé dans le système scolaire des groupes sociaux qui en auraient été exclus. En les écartant provisoirement des activités productives et en les coupant du monde du travail, l'école rompt la naturalité de la reproduction ouvrière fondée sur l'adaptation anticipée aux positions dominées, et les incline au refus du travail manuel et de la condition ouvrière $^{19}$. Les adolescents des quartiers de relégation sont de la sorte maintenus à l'école, tout en étant voués, par leur défaut de capital culturel, à un échec scolaire à peu près certain. Le hiatusentre l'avenir possible (entretenu par les discours sur la "démocratisation" scolaire) et l'avenir probable (dont ils font directement ou indirectement l'expérience) sape les fondements de l'autorité des enseignants. Cette illusion promotionnelle, dont parlent Sandrine Garcia et Franck Poupeau, c'est-à-dire le discours visant à reporter sur l'école les espoirs de promotion sociale, rend en effet 
l'école détestable aux yeux de ceux qui attendent d'elle ce qu'elle ne peut justement pas leur fournir (ou alors, seulement au rabais) : un travail, une place dans la société, une identité sociale. L'autonomie relative du système d'enseignement n'implique pas en effet qu'il puisse éradiquer les inégalités puisqu'il ne fait que les retraduire scolairement ${ }^{20}$. Cette illusion déçue s'est traduite par une banalisation de violences concrètes et quotidiennes - particulièrement au collège - qui expliquent que des agents, véritablement débordés dans leur travail, soient réceptifs à des formes de soutien, policier notamment, qu'ils récusaient auparavant.

Exclus ou en sursis dans le monde scolaire, surnuméraires dans un marché du travail auquel ils participent par intermittence, ces adolescents sont - pour une part non négligeable - ceux que Robert Castel appelle des "désaffiliés ", ces « inutiles au monde, qui y séjournent sans vraiment y appartenir. Ils occupent une position de surnuméraires, en situation de flottaison dans une sorte deno man's landsocial, non intégrés et sans doute inintégrables (...). Ils ne sont pas branchés sur les circuits d'échanges productifs, ils ont raté le train de la modernisation et restent sur le quai avec très peu de bagages ${ }^{21}$.

Ils tendent à se refermer - pour des temps et selon des modalités variables - sur un groupe de pairs avec qui ils partagent la même indignité sociale, culturelle et professionnelle. Le groupe les protège de la sorte des rappels à l'ordre des diverses institutions (école, missions locales, etc.), des autres adolescents (ceux qui ont un travail, réussissent scolairement) ou des jeunes filles (que la scolarisation prolongée "rend plus sensibles à la séduction qu'exerce la détention de capital culturel et/ou du capital économique et qui les détourne des charmes 'naturels' de la force physique et de la virilité $\$^{22}$ ). S'y bricole une identité faite de valeurs et de normes communes (musicales, vestimentaires, linguistiques, culturelles ${ }^{23}$ ) qui valorise les solidarités spatiales (plus que communautaires) autour du quartier, voire même de la cage d'escalier. Ces sociabilités mouvantes dessinent un monde de l'errance immobile en bas des tours, de l'ennui (omniprésent dans les chansons de rap), de la "glande ", des anecdotes cent fois racontées, déformées et amplifiées, des rumeurs, mais aussi celui d'une conscience de l'injustice nourrie par le racisme, les contrôles policiers à répétition, l'humiliation des pères, etc. A l'instar des sous-prolétaires algériens décrits par Pierre Bourdieu, le rapport au temps de ces adolescents ou jeunes adultes est celui de l'immédiateté et de la débrouille quotidienne ${ }^{24}$ : contrats d'intérim (bâtiment, manutention, sécurité, etc.) ; travail au noir et business, terme suffisamment flou pour caractériser un ensemble d'actions qui vont de l'échange non marchand de biens contre services, au petit deal, ou au recel. Ces pratiques sont à la fois des pratiques dominées (notamment parce qu'elles sont définies en négatif et tendent à fonctionner sur l'inversion du stigmate) et partiellement autonomes en ce sens qu'elles constituent des tentatives sans cesse renouvelées pour sauver symboliquement son honneur, ou gagner le respect ${ }^{25}$.

Dans des milieux populaires aujourd'hui traversés par des compétitions et des concurrences pour l'accès à des ressources rares - le travail non qualifié, le logement, les prestations sociales, etc. - qui exacerbent les tensions en leur sein, ces comportements achoppent vivement avec les systèmes normatifs des ouvriers précarisés et "prisonniers" de cités dont ces jeunes incarnent de manière particulièrement visible - et bruyante - le déclin collectif ${ }^{26}$. L'occupation permanente de l'espace public par ceux que Norbert Elias et John L. Scotson appellent la minorité des pires $^{27}$ rappelle à chaque instant la perte de statut social et de la fierté ouvrière afférente, vestiges d'un monde industriel en déclin. Elle génère un repli sur l'espace domestique 
et un malaise profond qui sera enregistré de manière tronquée par les sondeurs comme le «sentiment d'insécurité ». Ce malaise est redoublé par la disparition symbolique du groupe ouvrier en tant que groupe plus ou moins unifié et doté de porte-parole. En effet, l'individualisation de la condition salariale, en défaisant structuralement ce groupe, a détruit les dynamiques collectives au principe de son existence politique ${ }^{28}$.

(Dé)politisations populaires

L'observation des résultats électoraux dans les quartiers populaires français fait apparaître un désintérêt de plus en plus marqué pour les compétitions politiques. En témoignent les taux massifs d'abstention que l'on y observe et qui s'éloignent significativement des moyennes locales ou nationales. Henri Rey montre de la sorte dans une étude qu'il a menée dans 32 quartiers en convention politique de la ville que les taux d'abstention y sont de $52,1 \%$ en moyenne, soit 20 points de plus que la moyenne ${ }^{29}$. Certains quartiers de Seine-Saint-Denis dépassent quant à eux les $70 \%$ d'abstention, auxquels il faut ajouter 20 à $30 \%$ d'étrangers non communautaires qui $n$ 'ont pas le droit de vote et un bon nombre de non inscrits sur les listes électorales.

Il serait ambitieux de vouloir dresser ici une analyse exhaustive de la politisation populaire, ce qui ne va pas sans poser des problèmes épistémologiques ${ }^{30}$ (qu'est-ce qu'un comportement "politique»?) et demanderait une étude spécifique. Je me contenterai donc de suggérer quelques pistes en les reliant aux transformations morphologiques et sociales que j'ai décrites. J'aborderai successivement le militantisme communiste, la participation de la première génération d'immigrés à la politique et le rapport des jeunes sans avenir aux enjeux politiques.

Le parti communiste français (PCF) a longtemps tiré sa force de la chaîne de structures d'encadrement, qui partait de la vie la plus quotidienne des milieux populaires (dans les immeubles, les quartiers, les usines, etc.), pour arriver jusqu'aux pôles de pouvoir, locaux (municipalités) ou nationaux (parlement). Il assurait de la sorte une transmutation de faits sociaux (liés à l'expérience quotidienne) en faits politiques. De plus, en promouvant des cadres ouvriers, il donna longtemps l'image d'une unité représentants/représentés qui lui permit de revendiquer avec un certain succès le statut de "parti de la classe ouvrière", particulièrement bien implanté dans les bastions industriels du pays ${ }^{31}$. Le chômage massif d'une part, l'individualisation et la précarisation des statuts d'autre part vont toucher durement le PCF, au moment même où sa clientèle privilégiée (les ouvriers qualifiés) quitte les quartiers HLM pour s'établir dans des pavillons. Fragilisés dans leurs fiefs traditionnels alors que s'amplifient les désordres urbains (à Vaulx en Velin par exemple), les communistes vont se crisper sur la défense de ceux qui sont les plus proches de l'identité de classe qu'ils défendent, c'est-à-dire les ouvriers à statut (souvent français), opposés aux jeunes sans avenir professionnel (souvent d'origine étrangère). Ce hiatus va bloquer l'intégration de jeunes cadres issus des cités (et notamment du monde associatif) dans le parti ${ }^{32}$. La conséquence en sera une coupure entre communistes et jeunes des quartiers, qui se traduit au mieux par de l'indifférence au pire par de l'hostilité. Comme le soulignait un membre du bureau national du PCF :

"Avant, les loubards, on ne les comprenait pas non plus, mais comme il y en avait toujours un ou deux qui collaient des affiches pour nous, ils avaient au moins conscience qu'on était de leur côté.

Aujourd'hui, ce n'est plus le cas. Pour ces jeunes on ne représente plus rien». [Entretien, mars 2002]. 
Si le Parti a longtemps fonctionné comme une instance de socialisation politique des milieux populaires, il ne remplit plus ce rôle dans les cités périphériques françaises.

Les relations qu'entretiennent les immigrés maghrébins à la politique ont elles aussi été profondément transformées à la faveur des restructurations industrielles et de l'établissement durable en France. Pour les premières générations, exclues de la participation au jeu politique français ce rapport restait schématiquement structuré autour de deux pôles : la politique du pays d'origine et les luttes sociales dans les usines ou les foyers. L'intérêt des travailleurs immigrés pour la politique dans leur pays d'origine était lié aux luttes pour l'indépendance (FLN algérien par exemple) en même temps qu'à la perspective de rentrer au pays. Au fur et à mesure que s'éloigne la perspective du retour ${ }^{33}$ et que s'éteignent les feux de la décolonisation, cet intérêt s'estompe peu à peu, de même d'ailleurs que la force des organisations qui le portaient. Le second pôle de politisation des travailleurs immigrés en France s'est structuré lors des conflits sociaux et syndicaux. Leur concentration au plus bas de l'échelle sociale (comme OS) les a fragilisés. Premières victimes des restructurations qui les privent de leur emploi, ils vont de surcroît voir se déliter la fragile solidarité des travailleurs français avec qui ils entrent en concurrence, dans cette période de raréfaction des postes. Les grandes grèves des OS des usines Peugeot et Citroën entre 1982 et 1984 et la position plus qu'ambiguë de la CGT lors de ces conflits illustre bien ce phénomène.

La socialisation politique de leurs enfants va emprunter d'autres chemins que ceux du syndicalisme ouvrier, du militantisme communiste ou des luttes de libération. Sans statut dans le monde du travail (intérimaires à vie, jeunes n'ayant jamais travaillé, etc.), précarisés dans le monde industriel (CDD, rotation rapide d'un poste à l'autre, horaires décalés) ces jeunes peuvent difficilement connaître une insertion syndicale de type traditionnel. Il faut des conjonctures très particulières (comme la grève des travailleurs de Mc Donald à Paris en 2002) pour qu'ils accèdent à cet univers revendicatif, avec ses règles et ses codes. Il en va de même dans l'univers politique institué. Coupés, on l'a vu du parti communiste, ils ne se rapprochent pas davantage de ses concurrents. Le caractère lointain et abstrait des enjeux, l'absence de militants connus et reconnus dans leur quartier ne permettent pas de renverser leur sentiment d'incompétence politique collectif, et se traduisent dans la plupart des cas, par une position de retrait, d'indifférence, voire de méfiance. L'invective d'un jeune homme de Dammarie les Lys, lors d'un forum sur les violences policières quelques semaines après les élections présidentielles des 2002, à un parterre de militants associatifs, syndicaux et politiques résume bien cette coupure :

"Vous avez tous voté Chirac au deuxième tour! Vous êtes contents, c'est la démocratie! Mais moi Le Pen, je m'en fous. Il ne m'a jamais rien fait Le Pen! C'est pas lui qui tue nos frères! Ceux qui tuent nos frères, c'est Chevènement, c'est Sarkozy ${ }^{34}$.

Nombreux sont d'ailleurs ceux qui ne sont pas inscrits sur les listes électorales. Enfin, peu d'adolescents de seconde génération sont au fait des luttes politiques qui secouent le pays de leurs parents. C'est d'ailleurs ce qui explique leur faible attirance pour les groupes d'opposition qui peuvent exister en France, et particulièrement pour les groupes radicaux ${ }^{35}$. Les difficultés qu'ils éprouvent par exemple à parler de la situation algérienne expliquent pour partie le déplacement de l'engagement - notamment chez les filles - sur la question palestinienne. La solidarité avec les Palestiniens - qui se traduit par la création de comités, l'organisation de débats, d'expositions, de manifestations - s'explique, outre le travail d'entrepreneurs de mobilisation, par un 
sentiment diffus d'homologie de situation entre les Palestiniens là-bas et les jeunes immigrés ici : discrimination, racisme, contacts répétés avec les forces de l'ordre, etc. Ces derniers éléments sont fondamentaux pour comprendre les mobilisations que l'on observe depuis vingt ans dans ces quartiers. En effet, c'est autour de ces différents thèmes, qui renvoient à des questions de police et de justice, que se sont organisées les plus grandes manifestations politiques de ces populations.

De la marche pour l'égalité et contre le racisme (1983) à la création des jeunes arabes de Lyon et de banlieues (JALB) ou au mouvement de l'immigration et des banlieues (MIB), les « bavures » policières, les crimes racistes, la double peine ou les suicides en prison ont été le dénominateur commun de l'engagement ${ }^{36}$. Ceci n'a rien de surprenant, le militantisme en milieu populaire reposant sur l'expérience quotidienne plutôt que sur des cadres abstraits. Ce rapport au politique d'adolescent(e)s ou de jeunes adultes ne trouve toutefois que rarement des débouchés dans les arènes de la politique, dont ils ne partagent ni le langage, ni les principes de vision et de division du monde. La relation qui se noue alors entre professionnels et profanes repose simultanément sur un déni du politique pour les mobilisations qui remettent en cause le plus radicalement les règles du jeu ou les clivages légitimes, et sur la valorisation des initiatives les plus conformes. $C^{\prime}$ est ainsi que les mobilisations contre les violences policières par exemple, sont le plus souvent renvoyées à des registres explicatifs émotionnels ou instrumentaux ${ }^{37}$, alors que celles contre la "violence» en général trouvent un écho favorable au niveau institutionne ${ }^{38}$. Mais ce déni du politique dépasse largement la sphère des autorités locales et se retrouve avec la même acuité au sein des groupes ou des partis qui seraient objectivement les plus proches de ces populations. N'empruntant ni le vocabulaire, ni les thématiques, ni les registres d'action, ne partageant pas la même histoire des luttes que l'extrême gauche, les mobilisations de ces quartiers sont renvoyées à l'infrapolitique, au spontané, voire pire, au "communautarisme». Les échecs répétés des mouvements d'extrême gauche pour mener des actions autonomes dans ces quartiers, de même que leurs réticences à s'engager dans une lutte aux côtés d'associations confessionnelles montrent combien les économies symboliques qui structurent ces militantismes sont différentes, et la communication difficile. Les récents débats autour de la participation au forum social européen de Saint-Denis d'associations comme les jeunes musulmans de France, ou les querelles autour du soutien aux jeunes filles voilées exclues des établissements scolaires soulignent clairement ce malaise.

L'effondrement des structures de représentation des milieux populaires, leur désintérêt croissant pour la politique instituée et la disqualification dans l'espace politique légitime des luttes qu'ils peuvent mener constituent des facteurs clés pour comprendre l'évolution des catégories d'appréhension publique de la vie quotidienne des cités et le glissement vers une conception sécuritaire des rapports sociaux. En effet, ils font de ces univers, des univers objet, c'est-à-dire des univers qui ne sont plus en capacité de produire - avec une chance minimale de succès - une représentation d'eux-mêmes dans les luttes symboliques pour la division du monde. Ceci laisse le champ libre des représentations (politiques, médiatiques, institutionnelles, voire académiques) fortement marquées par un ethnocentrisme social qui s'ignore ${ }^{39}$. Appréhendant alternativement les milieux populaires sous l'angle du «manque », du « déficit » ou au contraire de "l'immoralité » et de la «dangerosité ", ces représentations font l'économie des rapports de forces structurant la production de normes et peuvent dès lors prétendre les imposer par le renforcement du contrôle et des structures de «normalisation». 
Des causes sociales du crime à la responsabilité individuelle du délinquant

Contrairement à ce que pourraient suggérer des formes de visions spontanées, les transformations morphologiques et sociales des quartiers populaires ne suffisent donc pas à expliquer mécaniquement l'intérêt croissant d'une fraction de la classe politique pour les "problèmes des banlieues", pas plus qu'elles ne permettent de comprendre les évolutions de leur mode de prise en charge publique. Il faut pour cela s'intéresser aux logiques endogènes du champ politique de même qu'aux relations qu'entretient ce dernier avec les médias et les professionnels de la sécurité.

L'attention des hommes politiques pour les questions de délinquance est récente. Elle date de la fin des année 1970, où sous le label «d'insécurité » s'opère pour la première fois - avec le rapport Peyrefitte notamment ${ }^{40}$ - une séparation entre le «crime » et la " peur du crime ». Cette rupture est décisive dans la mesure où les politiques, s'ils ne peuvent rien faire contre la délinquance (qui reste de la responsabilité exclusive de la police et de la justice), peuvent agir sur le «sentiment d'insécurité » de leurs administrés. C'est le point de départ de la spécialisation de certains élus et de la constitution de ce thème en bien politique.

Les rodéos automobiles et les affrontements avec la police de groupes de jeunes des Minguettes et de Vénissieux, durant l'été 1981, sont de la sorte souvent présentés comme la première manifestation importante de l'intérêt des pouvoirs publics pour les problèmes des cités périphériques. Les principales mesures prennent tout leur sens dans un contexte d'alternance (la victoire de François Mitterrand et de la gauche) et de relève du personnel politique. S'accordant sur l'idée que ces désordres sont induits par des causes sociales comme la précarité, le chômage ou la dégradation physique de l'habitat populaire, les politiques menées vont porter sur le développement social des quartiers, la prévention de la délinquance, l'amélioration du bâti et l'insertion des jeunes. Elles sont pour l'essentiel regroupées sous le label générique de politiques de la Ville et donneront lieu à la création à la fin de la décennie d'une Délégation Interministérielle à la Ville (DIV) et d'un ministère de la Ville. Elles reflètent en cela les sensibilités d'une gauche garante de la liberté, opposée à une droite historiquement partisane de la sécurité.

Pour autant, la question urbaine ne constitue pas à cette période une priorité de l'action gouvernementale et va échoir à des agents en position de relatif déclassement au sein des nouvelles élites d'Etat. Les différentes institutions de la politique de la Ville, leurs bailleurs (comme la Caisse des dépôts et consignations) et les expertises qu'elles vont mobiliser vont attirer des agents issus de la nébuleuse "modernisatrice » ${ }^{41}$. Largement impliquées dans les transformations de l'Etat dans l'après guerre, ces élites politico-administratives, dont les figures éponymes en politique furent respectivement Pierre Mendès-France et Michel Rocard arrivent au pouvoir dans une situation très marginale au sein du parti socialiste, par rapport aux autres courants, comme le CERES par exemple ${ }^{42}$. Elles vont donc se replier sur des chantiers périphériques de l'action gouvernementale et notamment la Ville. Important sur ce terrain leurs principes de rationalité et de rationalisation de l'Etat (logiques de projet, de territoire, de partenariat, etc.), elles vont jouer un rôle important dans la construction de catégories cognitives du problème, qui vont largement structurer son appréhension publique ${ }^{43}$. Ces approches, qui prennent le contre-pied des analyses en termes de domination et insistent sur les "handicaps" individuels des territoires et des populations, vont largement dépolitiser ce thème et poser les bases du consensus politique postérieur. 
Elles prennent toute leur mesure au début des années 1990, à la faveur de l'attention croissante portée par les médias aux embrasements sporadiques des banlieues ${ }^{44}$ et des transformations des relations entre hommes politiques et médias, dans le travail de définition symbolique des «problèmes sociaux $»^{45}$. C'est alors que les désordres urbains accèdent au statut de bien politique sur lequel vont s'opposer les professionnels de la politique. Et paradoxalement, les profits symboliques attachés à ce thème vont échapper à ceux qui avaient contribué à le faire émerger.

La victoire des partis de droite en 1993, marque un premier infléchissement: les problèmes des banlieues deviennent une question de police et de développement économique. Comme l'indique le premier rapport parlementaire sur la politique de la Ville, présenté par Gérard Larché, un sénateur de droite avant l'alternance : «il n'est pas étonnant (...) que la politique dite 'de la Ville', incriminant les erreurs de l'urbanisme et de l'aménagement, fermant encore trop les yeux sur des dérives sociales inacceptables, noyée par la bureaucratie et préoccupée d'abord par ses retombées médiatiques aboutisse aujourd'hui à un constat d'échec. A force d'accuser le béton de tous les maux, on a trop oublié les hommes. Or, sans responsabilisation des individus (...) il ne sera pas possible de restaurer l'équilibre - dans la différence assumée - de nos villes ${ }^{46}$. Cette responsabilisation de l'individu - liée à la doxa conservatrice - est au cœur des mesures gouvernementales : la loi d'orientation et de programmation sur la sécurité intérieure (LOPS), adoptée en 1995, vise à renforcer et à durcir la répression de la petite délinquance, alors que les différentes mesures de politique de la Ville insistent sur le développement par l'économie (zones franches, emplois aidés, etc.).

Le retour au pouvoir du parti socialiste en juin 1997, confirme cette évolution. Dans les concurrences internes au parti, les "modernisateurs » ont perdu toute l'influence politique qu'ils avaient conquise dans les années 1980-1990 et ce sont d'autres groupes qui vont se saisir de la question urbaine, devenue une question centrale. Les luttes politiques étant indissociablement liées à des luttes pour la définition du monde social, l'affaiblissement $\mathrm{du}$ rôle de ces élites d'Etat s'accompagne du déclin de leurs conceptions. L'approche urbaine globale qu'ils défendaient cède la place à une vision plus directement centrée sur la sécurité urbaine, érigée au rang de seconde priorité du gouvernement après l'emploi. Il n'y a d'ailleurs pas de ministre de la Ville dans le premier gouvernement Jospin. Il ne sera nommé qu'un an plus tard, en mars 1998, et restera condamné à une quasi inexistence tant symbolique que matérielle face à ses collègues de l'Intérieur et de la Justice. Les responsables socialistes insistent dès lors sur l'idée que « la première cause du crime serait le criminel lui-même ». C'est pour les partis de gauche, la fin de l'idée qu'il y a des causes sociales au crime: "on sait que la délinquance n'a aucune nature sociale et qu'elle relève de la responsabilité individuelle de chacun » rapportait ainsi Christophe Caresche, député socialiste de Paris ${ }^{47}$. Ces schémas présupposent alors que les adolescents des quartiers populaires auraient fait le choix facile, rationnel et durable d'un système de valeurs "délinquantes » contre celui de valeurs « conventionnelles ", où le travail reste central.

Il importerait alors de renchérir le coût de l'acte pour le délinquant en élevant la punition. Comme le déclarait Julien Dray, secrétaire national du Parti socialiste chargé de la sécurité, aux rencontres nationales sur la sécurité (Evry, 27 octobre 2001): "référons nous, pour une fois, aux préceptes des économistes néo-classiques: pour l'homoceconomicus rationnel, le prix de la possible punition doit excéder les bénéfices attendus du délit ». Ces conceptions insistent de la sorte sur la poursuite systématique de tous les 
délits et infractions. Jacques Chirac, président de la République déclarait ainsi : «nous avons des quantités de délinquants, notamment de jeunes délinquants qui n'ont même pas le sentiment de faire mal et qui agressent, il n'y a aucune suite donnée. Il est donc indispensable que l'on retienne le principe que toute agression, tout délit doit être sanctionné au premier délit $»^{48}$. L'effacement des oppositions droite/gauche dans la manière d'appréhender les illégalismes populaires et l'accent mis sur la responsabilité individuelle transforment l'économie de la punition. Ils sont le fruit d'une restructuration des processus disciplinaires antérieurs qui valide et autorise des systèmes de savoirs spécifiques.

Ces savoirs, essentiellement de type "béhavioriste», mettent l'accent sur les comportements "déviants", " antisociaux " et sur les "incivilités " des jeunes des quartiers populaires, dont ils font la cause de «l'insécurité » et le point de départ de «carrières » délinquantes ${ }^{49}$. Fortement influencés par les travaux de J.Q. Wilson et G. Kelling aux Etats-Unis, et notamment leur théorie des Broken windows ${ }^{50}$ (de la vitre cassée), ils insistent sur un continuum délinquant, qui partant d'actes insignifiants conduirait à la commission d'actes beaucoup plus graves, s'il n'était pas réprimé à temps. Après avoir servi de base à la réforme de zero tolerance de Rudolph Giuliani à New York et s'être acclimatés au Royaume-Uni avec les lois de Law and Order de Tony Blair, ces conceptions s'actualisent en France de manière particulièrement radicale dans la notion de "violences urbaines ", qui conduiraient graduellement de faits aussi hétérogènes que le vol de voiture, la dégradation d'une boîte à lettre, et l'impolitesse, à la criminalité organisée ou au terrorisme islamiste ${ }^{51}$.

Les multiples réfutations scientifiques ou empiriques de ces approches ${ }^{52}$ ne les empêchent pas de s'imposer dans le monde politique. Ce succès tient à la configuration dans laquelle ces conceptions s'inscrivent, à la position de ceux qui les énoncent et à la philosophie implicite qu'elles véhiculent.

D'abord, elles interviennent dans un contexte de déclin des expertises alternatives, qu'elles soient politiques (PCF, organisations de quartier et/ou issues de l'immigration) ou qu'elles émanent d'autres institutions, comme les services de prévention sociale, frappés de plein fouet par les conséquences de la désaffiliation. Cet affaiblissement autorise un renouvellement des cadres cognitifs de la question sociale, sous l'action d'agents intéressés à produire de nouvelles catégories plus conformes à leurs visions et à leurs intérêts. Ensuite, ces théories bénéficient de la légitimité et des positions d'autorité de ceux qui les portent. Occupant simultanément ou successivement des positions dans les champs académique (par des enseignements universitaires notamment dans des DESS ou des DU spécialisés en sécurité - par des publications d'ouvrages, etc.); politique (comme militants, membres de cabinets ministériels, conseillers techniques, etc.); administratif (par la participation aux écoles de formation, à des missions techniques, des rapports, etc.) et médiatique (comme experts mobilisés pour donner du sens, de la hauteur, à des séries de faits divers), ces agents multi-positionnés bénéficient des légitimités croisées de ces différents univers sociaux, qui tendent à fonctionner comme un multiplicateur de capital symbolique. Ils contribuent de la sorte à la mise en circulation et à la diffusion de nouvelles grilles d'analyse bien au-delà de leur espace d'élaboration ${ }^{53}$. Enfin, en enfermant les causes du crime dans l'observation des comportements criminels, ces savoirs fournissent des cadres théoriques qui paraissent immédiatement transposables en théories de la pratique, pour des hommes politiques soucieux de réformes ou d'améliorer l'action quotidienne des institutions. La focalisation de l'analyse sur les conséquences des illégalismes 
populaires opère également une réduction de la complexité sociale, qui favorise l'amnésie des responsabilités politiques dans les transformations structurelles du salariat. Pour le dire autrement, les discours sur les «violences urbaines» ou «scolaires » créent une politique de l'oubli et du silence sur la désaffiliation qui permet de fustiger les " mauvais pauvres », la « démission des familles populaires » et d'insister sur la nécessité de traitement policier de ces questions.

\section{Une gestion policière de ladésaffiliation?}

Les agences policières jouent un rôle particulier dans le processus d'élaboration, de diffusion et de naturalisation de ces systèmes de savoirs. Elles se caractérisent en effet par la capacité à produire des énoncés sur les illégalismes, les risques et les menaces dont le succès dépend d'un système d'échanges entre agents sociaux qui occupent des positions différentes dans et hors de l'Etat: gouvernement, hommes politiques, magistrats, institutions sociales et/ou d'encadrement, groupes de citoyens mobilisés, groupes criminels, etc. Les définitions de l'ordre (et du désordre) et de leurs modalités de régulation se modifient de la sorte en permanence, en fonction de l'évolution des relations entre chacun d'entre eux ${ }^{54}$. Les transformations du regard porté par la plupart des professionnels de la politique sur les illégalismes populaires, de même que les réelles difficultés que rencontrent les différentes institutions présentes sur ces quartiers (écoles, bailleurs sociaux, transporteurs publics, etc.) pour encadrer ces populations et leurs comportements, vont renforcer certains types d'expertises policières. Cette reformulation de la question sociale en question d'abord policière va donner à l'institution une place centrale dans la mise en forme du problème. Elle va modifier les équilibres antérieurs tant internes ${ }^{55}$ qu'avec d'autres espaces sociaux: justice, école, services sociaux, etc.

Ce qui ne va pas sans difficultés. En effet, si la police est valorisée, symboliquement comme en termes de crédits, les réponses qu'elle peut apporter restent ambivalentes. Les missions de «pacification sociale » n'intéressent pas beaucoup les policiers, dont la hiérarchie des normes est autre, plaçant au sommet de l'échelle le travail judiciaire voire le renseignement. La police est de factoune institution qui se caractérise, peut-être plus que d'autres, par le choix de ses missions et la manière dont elle va les exercer. C'est particulièrement le cas pour des branches "généralistes", comme la Sécurité publique. Ses policiers se rapprochent des street corner politicians décrits par W.K. Muir ${ }^{56}$, c'est-à-dire des agents qui vont choisir dans l'éventail des illégalismes, que la multiplication des règles et des règlements rend chaque jour plus nombreux, ceux qu'ils vont considérer ou pas. Cette hiérarchie des normes, jamais codifiée comme telle détermine, en dernière analyse, ce qui va être poursuivi dans l'activité ordinaire des agents.

Cette latitude d'action n'est pas forcément en phase avec les sollicitations extérieures. La question des groupes d'adolescents qui stationnent dans les halls d'immeubles jusqu'à des heures très tardives est à ce point de vue intéressante, puisqu'elle est le point de convergence de nombreuses plaintes, tant de la part de particuliers que d'agents institutionnels. Le décalage entre des demandes d'intervention relativement anodines mais répétées et la poursuite des délits limite l'enthousiasme des policiers pour intervenir. En effet, les demandes de régulation des petits désordres excèdent largement les capacités de la police et son savoir-faire (ou son savoir-être). Comme le rappelait un commissaire de police d'une grande ville du sud-ouest de la France : 
« Ici, il y a une spécialité, c'est le rugby, c'est-à-dire qu'on passe la balle à son voisin. Le problème c'est que la police est sur l'aile, au bord de la touche et qu'elle ne peut la refiler à personne!». [Commissaire principal de Sécurité publique, 44 ans. Entretien mars 2001].

Cette intervention solitaire, qui se réduit souvent à une répression sans délits, un contrôle sans infractions, reste de surcroît très difficile. Un chef de police de région parisienne résumait ainsi l'action de ses brigades anti-criminalité (BAC) dans les halls d'immeuble :

«S'ils trouvent quelque chose, arme, shit [haschich] ou autre, ils interpellent, mais sinon, ils se contentent de contrôles d'identité et les font partir en leur expliquant qu'ils font chier tout le monde ». [Commissaire divisionnaire de Sécurité publique, 53 ans. Entretien, mars 2001].

Dans tous les cas de figure, ces missions restent peu gratifiantes judiciairement et leur répétition instaure un fort climat de défiance entre forces de l'ordre et les groupes qu'elles contrôlent. Défiance qui trouve une traduction immédiate dans l'augmentation des outrages, voire des rebellions, qui passent de 11687 en 1974 à 43937 en 200157. Ces deux délits devenant d'ailleurs le plus souvent le seul chef d'inculpation possible dans ces situations ${ }^{58}$. Comme le soulignait un magistrat :

«On se rend compte que c'est le contrôle d'identité lui même - contrôlé et ordonné par l'autorité judiciaire - qui provoque l'apparition des délits. Au départ, on a une personne qui n'a rien fait, qui ne devait pas être contrôlée et qui au bout du compte se retrouve poursuivie par la justice pour un délit qui est provoqué directement par le contrôle lui même. C'est-à-dire en effet l'outrage, parce qu'il est parfaitement humiliant de se faire contrôler quand il n'y a aucune raison de l'être et qu'il est particulièrement humiliant de se retrouver avec les menottes ou emmené au poste parce qu'on a pas une carte d'identité dans la poche. Donc dans ces cas là, il y a un entraînement assez fréquent et assez légitime, je dirais, des personnes qui ne veulent pas sortir une carte d'identité, parce qu'on ne peut rien leur reprocher ou qui sortent leur carte d'identité mais contestent la légitimité de l'action policière. Et dans ces cas là, on voit que absence de contrôle, absence de volonté de l'institution judiciaire, la personne est systématiquement condamnée selon des procédures rapides et dans lesquelles elle n'est pas à même d'exercer ses droits à la défense ». [Juge d'instance. Entretien, mai 2002]

L'autorité judiciaire est ainsi enrôlée dans une logique d'ordre public fort différente de ses modes ordinaires de fonctionnement et sommée de prolonger l'action de la police par des sanctions. Les circulaires des gardes des Sceaux n'ont cessé de se multiplier depuis une dizaine d'années appelant à l'abaissement du temps de traitement de affaires, à la poursuite systématique de tous les délits, et à plus de sévérité.

On assiste de la sorte à une accélération du délai de traitement des petits délits. C'est l'objet du traitement en temps réel de la délinquance (TTR). Expérimenté au début des années 1990 au Parquet de Bobigny et généralisé par Elisabeth Guigou quant elle devint ministre de la Justice (1997), le TTR constitue l'une des transformations majeures du système pénal français. Son principe est simple : «toute affaire élucidée, crime, délit ou contravention de $5 e$ classe, doit faire l'objet d'un compte-rendu téléphonique immédiat au Parquet par le service enquêteur; toute affaire dont il est ainsi rendu compte doit faire l'objet d'un traitement immédiat par le Parquet $"{ }^{59}$. D'abord limité, ce principe s'est généralisé à l'ensemble des Parquets, dépassant $90 \%$ de l'activité de certains d'entre eux. Il vise à rapprocher les temps judiciaires des temporalités de commission de l'acte: «si la 
lenteur de l'institution judiciaire est stigmatisée, ce n'est pas tant parce qu'elle serait intrinsèquement mauvaise que parce qu'elle ne correspond plus à la réalité d'une société dont les rythmes sont tout autres ${ }^{60}$. Ce qui se joue ici est en fait un changement des modes d'évaluation de l'activité judiciaire, qui induit une transformation de son sens même. Philippe Mary expliquait au sujet du TTR que «ces dispositifs de justice rapprochée et accélérée font figure de fers de lance du managerisme qui commence à imprégner l'ensemble de l'administration de la justice pénale et comptent parmi les indicateurs les plus clairs de cette logique systémique, où la justice serait comprise non comme un système rationnel, mais à travers la rationalité du système ${ }^{61}$.

Dans le même temps, on observe une extension de la sphère pénale à des comportements qui n'étaient jusque-là pas poursuivis par la Justice (violences scolaires légères, fraudes dans les transports en commun, et plus largement les «incivilités »). C'est l'objet de la 3e voie judiciaire, dont l'ambition affichée est de réduire les classements secs. Cette inflation pénale se traduit par la naissance, en amont de la chaîne pénale de toutes les procédures de médiation pénale, de conciliation, de réparation, qui s'effectuent dans les maisons de justice et du droit (MJD).

Enfin, on note un durcissement des peines prononcées pour les petits délits. La sévérité des chambres de comparution immédiate par rapport aux juridictions ordinaires est à cet égard particulièrement emblématique. Les transformations de la Justice des enfants, où on observe un accroissement massif des réponses pénales, au détriment des réponses éducatives, plus longues à mettre en place, sont un exemple significatif de ce mouvement ${ }^{62}$.

Ce durcissement observable dans le champ judiciaire est également manifeste dans les méthodes d'intervention de la police. Certains policiers eux-mêmes n'hésitent pas à dénoncer une militarisation des rapports qu'illustrent les attitudes (le " saute dessus », les opérations "coup de poing ") et les tenues adoptées par les unités spécialisées qui travaillent dans ces quartiers : combinaisons noires, assorties de nombreux accessoires (tonfas, bombes lacrymogènes de grande taille, etc.), casques, armement (flash-balls ${ }^{63}$, fusils à pompe). Le vocabulaire emprunte de plus en plus au registre guerrier. Des policiers comme Richard Bousquet, commissaire divisionnaire et responsable du syndicat des commissaires et hauts fonctionnaires de la police nationale (SCHFPN, majoritaire) parlent de « logique de guerre », de « zones de sécurité » qui entourent des cages d'escalier, "d'armée en ordre de marche disposant d'une logistique efficace », de " fantassins de la drogue ${ }^{64}$, etc. Les logiques d'action sont à l'unisson. Justifiant l'emploi des unités mobiles spécialisées (UMS), le préfet d'un département de petite couronne parisienne expliquait :

«C'est un outil majeur d'endiguement des violences urbaines dans le département. Dès qu'il y a une rixe entre bandes et qu'un équipage [de police] est en difficulté, j'ai trente mecs taillés qui peuvent se porter sur place en 10-15 minutes. Ça les calme... et c'est plus efficace qu'un CRS, qui ne connaît pas le terrain et qui se déploie à l'aveuglette (...). L'îlotage ne sert à rien s'il n'est pas appuyé par la cavalerie. L'îlotier n'arrête personne et s'il ne se sent pas épaulé, il rase les murs. Il est donc hors de question que je me départisse de ma cavalerie ». [Préfet, ancien délégué interministériel à la Ville et au Développement Social Urbain, 53 ans. Entretien, janvier 1999].

Le chef de la sûreté urbaine d'une circonscription située dans l'un des trente départements classés « très sensibles » décrivait pour sa part son travail ordinaire dans « ses » cités : 
"C'est le Kosovo, là-bas. On est en mission de pacification. Il faut tenir les hauts, comme les militaires tiennent les crêtes... On assiste à une surenchère dans le matériel utilisé. On ne se sert plus du flash-ball, qui était le nec il y a 5 ans. Les gardiens ont des grenades de désencerclement, qui sont des grenades à fort effet de souffle, et ils utilisent des fusils à pompe. Les balles sont certes en caoutchouc, mais pour le policier, le principal geste est fait : braquer quelqu'un avec un fusil à pompe. Avant, ces armes collectives ne sortaient pas des armureries, où alors, pour des missions très ponctuelles et encadrées. (...) Cela vient des consignes de tenir le terrain à tout prix, même en sous effectif. Et il faut le tenir, mais bon ». [Commissaire divisionnaire de Sécurité publique, 51 ans. Entretien, avril 1999].

Ceci dit, les consignes ne suffisent pas à justifier ce durcissement, qui découle également de motifs plus structurels, au premier rang desquels la jeunesse des unités d'intervention. Celle-ci s'explique, outre les sélections physiques nécessaires pour y entrer par le fort turn over qu'elles connaissent. Les policiers les plus expérimentés les désertent en effet grâce à leur ancienneté qui leur permet de demander des mutations dans des services plus "tranquilles» ou des rapprochements géographiques de leur région d'origine. Elles restent donc le plus souvent dépourvues "d'anciens» qui pourraient inculquer des savoir-faire opératoires et donner quelques clés de décryptage d'une situation incompréhensible à beaucoup, particulièrement en l'absence de formation adéquate. En effet, peu assurés professionnellement, ces jeunes policiers, souvent issus de petites villes de province, sont socialement très éloignés des cités et de leurs habitants, qu'ils soient où non d'origine immigrée. D'où leur malaise persistant à intervenir dans des grands ensembles dont ils ne connaissent ni les codes ni le fonctionnement, qui se traduit à la fois par une peur pour intervenir et surtout par une application restrictive du métier policier et l'absence de distance au jeu qui caractérise des policiers plus expérimentés et en affinité avec leur terrain.

Les interactions quotidiennes avec les groupes de jeunes prennent la forme de confrontations ritualisées où il s'agit de sauver l'honneur du groupe, voire de laver virilement les affronts. Dans cette relation de rivalité mimétique, sans doute jamais aussi nette que dans ce commentaire d'un policier à un adolescent : " toi y'a marqué Lacoste, moi y'a marqué Police $"^{65}$, toute défaite symbolique ou physique de l'un est vue comme une victoire de l'autre. Ceci explique les attroupements systématiques lors des contrôles voire les "caillassages", auxquels répondent d'inutiles vérifications d'identité à répétition, des intimidations, des humiliations, voire les coups. Pour les groupes de jeunes, il s'agit de "mettre la pression»- pour reprendre une expression souvent entendue en entretien - sur les policiers en leur faisant comprendre qu'ils sont en infériorité numérique et donc que le rapport de force est en leur défaveur. Pour les policiers, à l'inverse, l'enjeu est de montrer qu'ils sont les maîtres de l'espace public, quitte à faire un usage illégitime de leur monopole de la violence légitime ou à jouer sur les ressources d'autorité - morales et juridiques notamment - que leur confère leur statut ${ }^{66}$.

Pour autant, le durcissement des modes d'action et des relations, de même que l'augmentation du nombre de poursuites pour outrages ne résolvent pas la question initiale, celle des petites nuisances qui avaient déclenché ce type d'interventions.

L'enrôlement policier des services sociaux et éducatifs

Les policiers confrontés au quotidien à ce type de contradictions sont alors d'autant plus portés à s'investir dans les structures «partenariales », comme les Contrats Locaux 
de Sécurité (CLS), qu'ils ont pratiquement et symboliquement tout à y gagner. En effet, engager d'autres agents sociaux dans le contrôle et la normalisation de ces comportements déviants est l'une des solutions les moins coûteuses et les plus efficaces pour répondre à des troubles qu'ils sont incapables de résoudre, pour des raisons tant internes (priorités policières, « inversion hiérarchique ») que liées à leurs prérogatives (nécessité de constater un délit, déplacement des problèmes, de quelques mètres parfois en cas d'opérations intensives de police).

C'est ainsi qu'on assiste en France à un travail d'enrôlement policier des autres institutions, soit direct dans le cas des bailleurs sociaux, des transporteurs publics, voire des municipalités, qui développent leurs propres forces de sécurité ; soit indirect, pour l'école, les ANPE (agence nationale pour l'emploi), les missions locales d'insertion, etc., sommées de fournir des informations sur les adolescents qu'ils suivent ou côtoient. Cette collaboration repose sur le décloisonnement de l'information entre "partenaires". L'échange "dans le respect des déontologies réciproques" de données personnelles précises, sur des individus qui « posent problème » est vu comme l'une des clés du succès de l'action publique locale. C'est ce qu'on appelle dans les arènes où il se déploie le « secret partagé ». S'il est loin d'être unique, l'exemple du CLS de Chalon sur Saône - dont l'actuel ministre de la Justice est maire - est particulièrement emblématique :

«Il faut ici parler de traçabilité. Le constat est sévère. Tous les acteurs ont peu ou prou connaissance des noms et prénoms qui, dossier après dossier, groupe de travail après groupe de travail, reviennent dans l'actualité de notre CLS. A ceci près que le cumul de ces 'signalements' n'est centralisé nulle part. (...) Avec l'autorisation des co-signataires, [il faut] dresser l'état nominatif des familles et des fratries qui, sur l'année 2000 et sur l'année 2001 sont revenus régulièrement dans l'actualité et dans le suivi par les opérateurs principaux du CLS. Dix, vingt, cinquante familles, il est nécessaire de pouvoir dresser cette liste en croisant les données de la police nationale, de la police municipale, des services de la CAF, des services de l'Education Nationale, des bailleurs sociaux, de la ville, de la sous-préfecture. (...) Il faut alors dresser par famille, un 'bilan d'activité'. Qui travaille? Où? Qui ne travaille pas? Qui est scolarisé ? Qui suit la famille? Qui en rend compte à qui ? Qui connaît la situation? Qui intervient? Qu'a demandé la famille? Le mineur? Qu'ont déjà demandé les institutionnels? Ce bilan nécessaire, donnera l'état de la situation et désormais, cette famille ou cette fratrie aura l'intime conviction que quelque chose s'est mis en marche et que désormais, on ne peut plus ' jouer' entre les lignes et opposer telle ou telle administration à tel ou tel autre décideur $"{ }^{67}$.

Ce décloisonnement de l'information est un processus de divulgation dont l'ambition est de ruiner les différentes personnalités ou facettes que peut présenter un individu à différentes institutions. Il renvoie aux mécanismes du secret d'initiés décrits par Erwing Goffman : «les différentes images de lui-même qui lui sont habituellement renvoyées à tous les niveaux de son entourage finissent ici par se retrouver réduites, derrière son dos, à une seule $»^{68}$.

Mais cet échange reste profondément inégalitaire, puisque la police en conserve très largement le leadership, y compris d'ailleurs vis-à-vis de la justice. Comme le rappelle ce commissaire de police :

« Au GLTD [groupement local de traitement de la délinquance], on arrive avec la liste des gamins qui foutent la merde sur le quartier, et le juge dit ah! Mais on ne peut pas 
travailler si on donne des noms, etc. J'ai répondu que nous, on était pas là pour perdre notre temps et que s'ils ne voulaient pas travailler, nous on se retirait ». [Commissaire principal de Sécurité publique, 44 ans. Entretien, mars 2001].

Ce «partenariat » confère aux policiers une position de centralité assez nouvelle dans la régulation de comportements qualifiés publiquement de déviants, qui étaient auparavant pris en charge par d'autres institutions sociales ou sur d'autres modes ${ }^{69}$. Ce qui faisait dire non sans humour à un adolescent régulièrement confronté à la police :

«Maintenant, la BAC [brigade anti-criminalité], quand elle nous tape dessus, elle nous appelle par notre prénom » [Entretien, mars 2001].

La prégnance de l'expertise policière transfigure les formes de traitement de ces phénomènes. Murray Edelman rappelle en effet que les bureaucraties ont tendance « à construire les problèmes comme justifications des solutions qu'elles proposent $»^{70}$. L'habitus professionnel des policiers, qui place au sommet de la hiérarchie des normes le judiciaire - ce dont témoigne ce vieux leitmotiv : «nous ne sommes pas des assistantes sociales" - valorise la coercition. Les arrestations et les poursuites sont la pierre angulaire de leurs pratiques professionnelles. Et même si beaucoup de policiers sont conscients que les condamnations ne suffisent pas à faire disparaître le jeunes délinquants qu'ils côtoient, ils restent prisonniers d'une grille de lecture du monde fortement solidifiée : celle de leur institution et de ses fonctions sociales. Ceci se traduit par une naturalisation de la délinquance - qu'attestent les qualificatifs de mineur délinquant, de voyou, de malfaisant, etc. - au détriment de visions qui resituent l'acte délinquant dans une histoire de vie plus complexe, où il voisine avec de multiples autres insertions (scolaire, familiale, affective, professionnelle, etc.).

Ces antagonismes cognitifs pèsent lourdement sur les formes de solutions publiques qui vont être envisagées. La désaffiliation et son cortège de maux se transforment de la sorte en "délinquance des mineurs"; en "violences urbaines" ou en "violences scolaires ", autant de "problèmes" administrativement constitués appelant des « réponses» dans lesquelles la police occupe un rôle privilégié. Les standards, les formats policiers - même s'ils suscitent parfois des résistances - tendent ainsi à devenir le prisme pertinent d'appréhension de certaines populations. Ils s'imposent lentement dans des espaces sociaux où ils n'avaient pas cours auparavant. Comme le signalent Richard V. Ericson et Kevin D. Haggerty : "il n'y a pas de limites à la participation de la police dans la construction et la gestion des problèmes sociaux.[La police]fabrique les savoirs dont les autres institutions ont besoin dans la gestion des risques des populations particulières dont elles sont responsables $»^{71}$. C'est parfois le cas à l'école. Un proviseur de lycée de région parisienne m'expliquait de la sorte:

" Avec la police, cela se passe également très bien. Les établissements n'hésitent plus à faire appel à elle quand il y a un incident. Inversement, la police fait appel aux établissements quand elle veut interpeller quelqu'un: on communique l'adresse, on montre les photos, etc. C'est une collaboration durable qui s'est instaurée. Les réticences diminuent chez les chefs d'établissement; il se diffuse une mentalité qui fait tache d'huile : ils se disent, si mes collègues le font, il n'y a pas de raisons que je ne le fasse pas moi-même. Ailleurs, là où il n'y a pas eu de CLS, c'est une surprise. Un collègue s'étonnait que la police entre dans mon établissement, en uniforme de surcroît. Il m'a dit que chez lui ce serait l'émeute et que les profs se mettraient en grève. Ils restent encore très marqués par une mentalité 68 et pour eux les policiers, c'est les CRS ». [Entretien, mars 2001] 
Les ressorts de cette "collaboration durable", que l'on retrouve également dans d'autres institutions, comme la prévention spécialisée, l'animation socio-culturelle, etc. sont à rechercher dans la situation de porte-à-faux dans laquelle se retrouvent placés les agents subalternes de l'Etat (ou des collectivités locales), particulièrement ceux qui sont chargés de remplir les fonctions dites "sociales". Ceux-ci sont obligés de compenser sans en avoir les moyens les effets et les carences les plus intolérables de la logique du marché et des mutations économiques de ces vingt dernières années. La contradiction entre ces missions démesurées et la confrontation effective avec les populations les plus démunies économiquement et culturellement ne peut dès lors être résolue qu'au prix du sacrifice - et de l'exclusion - des éléments perturbateurs, qui mettent en péril les faibles chances d'amélioration du destin social collectif. C'est donc la police - et la justice - qui vont être chargées de résoudre la question centrale que pose Robert Castel: "que faire d'individus qui soulèvent des problèmes inextricables parce qu'ils ne sont pas à leur place, mais qui n'ont nulle part de place dans la structure sociale ${ }^{72}$. Les poursuites systématiques, l'incarcération ou la mise à l'écart dans des structures spécialisées (unités éducatives à encadrement renforcé [UEER], centres éducatifs renforcés [CER], centres éducatifs fermés) deviennent de la sorte le mode naturel de régulation de ces «surnuméraires».

Si ces mesures sont politiquement très fonctionnelles, particulièrement en période électorale, parce qu'elles permettent aux gouvernements successifs de s'exonérer des conséquences de leurs politiques et qu'elles viennent redonner force au mythe de la souveraineté du politique, battu en brèche en matière économique et financière notamment ${ }^{73}$, elles posent problème à moyen et à long terme.

La police n'a en effet pas les moyens de juguler la petite délinquance, qui constitue bien souvent - avec le travail intérimaire, les allocations sociales le travail au noir - l'un des seuls modes de (sur)vie de petits groupes exclus durablement du circuit des échanges productifs. Elle peut arrêter autant de revendeurs de drogue qu'elle veut, il existe une véritable armée de réserve de jeunes gens prêts à les remplacer ${ }^{74}$. C'est d'ailleurs ce qui explique ce sentiment de "puits sans fonds " qu'expriment plusieurs policiers de Sécurité publique en entretien. Comme le soulignent les études menées outreatlantique rien ne montre que l'augmentation du nombre de policiers sur le terrain ou que les stratégies intensives de police aient été responsables du déclin de la délinquance aux Etats-Unis ${ }^{75}$.

Ensuite, l'incarcération - dont la France vient d'atteindre un record historique avec 60 513 détenus en mai 2003 - reste marquée par de très forts taux de récidive. Le passage par l'enfermement renforce en effet le stigmate initial sur les marchés scolaire, de l'emploi, voire sexuel, rendant l'insertion plus difficile encore et la poursuite d'activités illicites plus plausible. Par un effet d'inversion, il tend également à devenir au sein du groupe de pairs un brevet de gloire qui enferme dans un rôle social de délinquant. De la même manière, la saturation de la présence policière dans certains quartiers creuse un fossé d'incompréhension entre leurs populations - et notamment les plus jeunes - et les institutions. Elle radicalise et durcit les positions de chacun, ce qu'illustre la multiplication des violences policières illégitimes observées ces derniers mois, à Poissy, Saint-Denis, Dammarie les Lys, Nîmes ou ailleurs ${ }^{76}$.

Ce court circuit sécuritaire est d'autant plus préjudiciable, qu'il repose sur des présupposés erronés. La sécurité n'est pas le contraire de l'insécurité, mais son double. Il faut insécuriser pour sécuriser, et toute procédure de sécurisation insécurise ${ }^{77}$. La 
fuite en avant dans le maelström sécuritaire génère des représentations du monde où tout devient menaçant et où chaque incertitude se mue en peur. Ce mouvement consolide le racisme et la méfiance à l'encontre de ceux qui tendent à devenir des «nouvelles classes dangereuses $»^{78}$ et rend, par là même, leur marginalisation plus profonde. Il constitue à l'évidence une impasse, sauf à admettre que le creusement des inégalités et l'exclusion de certains groupes sociaux ne relèvent que d'une prise en charge policière. C'est-à-dire à accepter la doxa conservatrice qui limite les fonctions régaliennes d'un Etat au maintien de l'ordre social et subordonne l'ensemble de ses activités intellectuelles, administratives et politiques à cette fin.

\section{NOTES}

1. . Elias N., Qu'est-ce que la sociologie ?, Paris, Ed. de l'aube, 1991, pp. 154 et suiv.

2. . Lenoir R., « Objet sociologique et problème social », in Champagne P., Lenoir R., Merllié D. et Pinto L. Initiation à la pratique sociologique, Paris, Dunod, 1996, p. 77.

3. . Entre 1955 et 1975, deux millions de logements sociaux ont été construits en France. Sur l'évolution de la question du logement depuis la seconde guerre mondiale, voir Flamand J.-P., Loger le peuple : essai sur l'histoire du logement social en France, La Découverte, Paris 1989 et Bachmann C. et Le Guennec N., Violences urbaines. Ascension et chute des classes moyennes à travers cinquante ans de politique de la ville, Paris, Albin Michel, 1996, particulièrement la troisième partie, pp. 105 et suiv.

4. . Seys B., " L'évolution de la population active ", INSEE Première n434, mars 1996. 5. . A partir d'une enquête en banlieue parisienne, Olivier Masclet montre à la fois les réticences des familles ouvrières à intégrer ces grands ensembles et l'exclusion des familles immigrées qui n'ont pu y accéder qu'à la faveur de leur désertification - et décadence - progressive. Voir Masclet O., La gauche et les cités. Enquête sur un rendezvous manqué, Paris, La dispute, 2003.

6. . Bourdieu P., Les structures sociales de l'Economie, Paris, Seuil, 2000, particulièrement pp. 113 et suiv.

7. . Il convient de manier les catégories «français » et « immigrés » avec prudence. En effet, elles ne reposent pas sur des catégories objectives désignant un groupe social, mais au contraire sont un perpétuel enjeu de luttes, à l'intérieur et à l'extérieur du groupe. Elles tendent de surcroît à agréger sous un même label des situations sociales, des statuts et des conditions hétérogènes, qui opposent les individus plus qu'elles ne les rassemblent. Il en va de même pour les représentations statistiques d'Etat, qui loin d'être neutralisées engagent des présupposés que l'on ne peut négliger lorsqu'il s'agit de distinguer statistiquement qui fait partie de la communauté nationale ou non. Pour autant, en l'absence d'indicateurs alternatifs, c'est sur ces taxinomies que sont basées les différentes données socio-morphologiques sur les « immigrés » utilisées infra. Pour l'INSEE, « est considérée comme immigrée toute personne résidant en France née à l'étranger et se déclarant de nationalité étrangère ou française par acquisition ». En 1999, la population immigrée de 15 ans ou plus vivant dans des ménages s'établit à 3 
800000 personnes. Près de $30 \%$ d'entre elles ont pris la nationalité française. (Thave S., « L'emploi des immigrés en 1999 », INSEE Première n717, mai 2000).

8. . En 1996, le parc social abrite près d'un ménage immigré sur trois (un sur deux pour les immigrés originaires du Maghreb), contre moins de un sur six pour l'ensemble des ménages. Boëldieu J. et Thave S., « Le logement des immigrés en 1996 », INSEE Première $\mathrm{n}^{\circ} 730$, août 2000 .

9. . Chardon O., «Les transformations de l'emploi non qualifié depuis vingt ans », INSEE Première $n^{\circ} 796$, juillet 2001.

10. . Thave S., «L'emploi des immigrés en 1999 », op. cit.

11. . Cézard M., « Les ouvriers », INSEE Première $n^{\circ}$ 455, mai 1996.

12. . Chardon O., "Les transformations de l'emploi non qualifié depuis vingt ans », op.

cit.

13. . Sur les comportements de ces groupes, voir Mauger G. et Fossé-Poliak C., « Les loubards ", Actes de la recherche en sciences sociales n50, novembre 1983.

14. . Voir notamment Willis P., "L'école des ouvriers ", Actes de la recherche en sciences sociales $n^{\circ} 24$, novembre 1978.

15. . Sur les transformations de la structure de l'emploi industriel et ses effets sur les adolescents des quartiers populaires, voir Pialoux M., « jeunesse sans avenir et travail intérimaire ", Actes de la recherche en sciences sociales, n²6-27, mars-avril 1979, pp. 19-47.

16. . « Dans le commerce, des emplois peu qualifiés ont accompagné l'essor de la grande distribution : les caissiers, les vendeurs en alimentation et les employés de libre-service sont 273000 de plus qu'il y a 20 ans. (...) Dans le domaine de l'entretien, les employés de nettoyage de bureaux ou de locaux industriels sont 117000 de plus qu'en 1982. De même, les professions d'agents de sécurité ou de surveillance se sont développées. (...) L'essor de la restauration et notamment de la restauration rapide a bénéficié aux emplois de serveurs, aides cuisiniers ou plongeurs, dont les effectifs ont augmenté de plus d'un quart. Au total, les professions non qualifiées en essor ont vu leurs effectifs progresser d'un million en 20 ans. En 2001, ces emplois représentent la moitié de l'emploi non qualifié, contre moins d'un tiers en 1982 ». Chardon O., « Les transformations de l'emploi non qualifié depuis vingt ans », op. cit.

17. . Les travaux qu'a pu mener Philippe Bourgois sur les dealers de crack à New York sont très éclairants pour comprendre comment ces normes et ces valeurs s'opposent en tous points à celles qui ont cours dans les nouveaux emplois déqualifiés. Voir Bourgois P., En quête de respect. Le crack à New York, Paris, Seuil, 2001, 327 pages, particulièrement chapitres 4 et 5 .

18. . Les enfants des ménages immigrés longeant en HLM quittent tardivement le foyer parental : 48\% des jeunes de 20 à 34 ans vivent encore avec leurs parents, soit deux fois plus souvent que la moyenne. Boëldieu J. et Thave S., « Le logement des immigrés en 1996 », op. cit.

19. . Sur ce sujet, voir Beaud S. et Pialoux M., Retour sur la condition ouvrière, Paris, Fayard, 1999, particulièrement la seconde partie « Le salut par l'école » et Grignon C., L'ordre des choses. Les fonctions sociales de l'enseignement technique, Paris, Editions de Minuit, 1971.

20. . Poupeau F., "Professeurs en grève ", Actes de la recherche en sciences sociales, n¹36-137, mars 2002, pp. 83-94 et Garcia S. et Poupeau F., «Violences scolaires : la faute à l'école ? » in Bonelli L. et Sainati G (dir.), La machine à punir. Pratiques et discours sécuritaires, Paris, L'Esprit frappeur, 2001, pp. 125-126. 
21. . Castel R., Les métamorphoses de la question sociale. Une chronique du salariat, Paris, Gallimard, 1999, pp. 665-666.

22. . Mauger G., « La reproduction des milieux populaires en 'crise' », Ville - Ecole Intégration $\mathrm{n}^{\circ} 113,1998$.

23. . Lepoutre D., Cœur de banlieue. Codes rites et langages, Paris, Odile Jacob, 1997.

24. . « Parce qu'ils ne peuvent fournir ce minimum de sécurité et d'assurances concernant le présent et l'avenir immédiat que procurent l'emploi permanent et le salaire régulier, le chômage, l'emploi intermittent et le travail comme simple occupation interdisent tout effort pour rationaliser la conduite économique en référence à une fin future et enferment l'existence dans la hantise du lendemain, c'està-dire dans la fascination de l'immédiat ». Bourdieu P., « Les sous prolétaires algériens ", Agone n²6/27, 2002, p. 205 [Les temps modernes, décembre 1962]. 25. . Bourgois P., En quête de respect, op. cit.

26. . Beaud S., « L'école et le quartier ", Critiques sociales n5-6, 1994, pp. 13-46. Olivier Masclet montre pour sa part le « déshonneur », la « honte » des pères immigrés algériens devant la déviance de leurs fils : « l'arrestation des 'fils indignes' (...) est plutôt le signe insupportable du fossé qui s'est creusé entre eux », entre ouvriers stables et sous-prolétaires, entre « ouvriers respectables » et « jeunes sans avenir ». Masclet O., « Les parents immigrés pris au piège de la cité », Cultures \& Conflits $n^{\circ} 46$, 2002, pp. 147-173.

27. . Elias N. et Scotson J.L., Les logiques de l'exclusion, Fayard, Paris 1997 [1965], pp. 158 et suiv.

28. . Thompson E. P., La formation de la classe ouvrière anglaise, Paris, Gallimard - le Seuil, 1988 [1963].

29. . Rey H., Etude pour le compte de la délégation interministérielle à la ville, rapport dactylographié, 2001.

30. . Sur ce sujet voir Mauger G., « La politique des bandes », Politix n¹4, 1991.

31. . Voir notamment Pudal B., Prendre parti. Pour une sociologie historique du PCF, Paris, Presses de Science-Po, 1989.

32. . C'est le rendez-vous manqué entre le PCF et les jeunes des cités que décrit de manière très détaillée Olivier Masclet dans La gauche et les cités, op. cit.

33. . Sayad A., La double absence. Des illusions de l'émigré aux souffrances de l'immigré, Paris, Seuil, 1999, 439 pages.

34. . Ministres de l'Intérieur français, respectivement de gauche et de droite.

35. . Khaled Kelkal, un jeune de Vaulx en Velin impliqué dans les attentats de 1995 en France constitue de ce point de vue une exception due à l'implication forte de sa famille dans les questions algériennes. Son père était à cette période imam en Algérie et très engagé dans l'islam politique.

36. . Pour un historique, voir Abdallah M.H., J'y suis, j'y reste ! Les luttes de l'immigration en France depuis les années 60, Paris, Reflex, 2001,160 p.

37. . Le préfet de région commentait ainsi les mouvements consécutifs au décès d'un adolescent, tué par un policier à Toulouse en décembre 1998 : « ces casseurs ne sont pas sans liens avec les 19 personnes que nous avons arrêtées récemment pour des casses chez des commerçants. Au-delà de l'émotion légitime ressentie après la mort du jeune Habib, on peut voir dans les mouvements du Mirail une réaction à ces arrestations qui n'ont certainement pas fait plaisir à tout le monde, en mettant un coup d'arrêt à toute sortes de trafics ». La Croix, 18 décembre 1999. Sur l'attitude de la municipalité et de la préfecture lors de la mobilisation d'un quartier de Dammarie les Lys durant l'été 2002, 
autour de l'association Bouge qui bouge et du Mouvement de l'Immigration et des Banlieues (MIB), voir Vacarme $\mathrm{n}^{\circ} 21$, automne 2002.

38. . L'exemple du mouvement Stop la violence, lancé à la suite de la mort de Stéphane Coulibaly à Bouffémont, dans le Val d'Oise, le 14 janvier 1999 est emblématique. Parti de la volonté d'un certain nombre d'adolescents de rétablir l'image de leur camarade, présenté dans les médias comme un délinquant, ce mouvement - encadré dès le début par un journaliste de Nova et un conseiller municipal socialiste (David Assouline, adjoint au maire du XXe arrondissement de Paris) - va connaître une reconnaissance rapide des pouvoirs publics et des médias. Fondé sur un manifeste condamnant « la violence des banlieues ", il cadre parfaitement dans le fond et dans les formes avec les catégories pertinentes du moment dans le jeu politique. Des émissions spéciales on lieu à la radio, Canal + tourne un documentaire, diffusé en mai, et surtout, des représentants sont invités à grand renfort de publicité aux Rencontres nationales des acteurs de la prévention de la délinquance à Montpellier, en mars 1999. Le ministre de la Ville, Claude Bartolone les reçoit, alors que Jean-Pierre Chevènement, ministre de l'Intérieur, leur adresse un message d'encouragement, avant de donner des consignes aux préfets pour favoriser le développement de groupes locaux.

39. . Claude Grignon et Jean-Claude Passeron parlent même de racisme de classe, entendu comme « certitude propre à une classe pour monopoliser la définition culturelle de l'être humain et donc des hommes qui méritent d'être pleinement reconnus comme tels ». Ils précisent également que cette certitude " habite de vastes secteurs des classes dominantes, et pas forcément les plus traditionnels ou les plus élitistes ». Grignon C. et Passeron J-C. Le savant et le populaire. Misérabilisme et populisme en sociologie et en littérature, Paris, Gallimard-Le Seuil, 1989, p. 32. 40. . Comité d'études sur la violence, la criminalité et la délinquance, Réponses à la violence, Paris, Presses Pocket, 1977.

41. Pour la nébuleuse modernisatrice, et les transformations des manières de dire et de faire l'Etat durant les «trente glorieuses », voir Kuisel R. F., Le capitalisme et l'Etat en France. Modernisme et dirigisme au XXème siècle, Paris, Gallimard, 1984, 477 pages. 42. . Le CERES était un courant d'orientation marxiste dirigé par Jean-Pierre Chevènement, qui rédigea notamment le programme du parti socialiste de 1980. Lire à ce sujet les doléances et l'amertume exprimées par Serge July et Michel Marian, dans «Surpris, soufflés hors du coup... », Esprit n¹0-11, octobre-novembre 1981, pp. 196-210.

43. . L'ouvrage de Jacques Donzelot et Philippe Estèbe (L'Etat animateur. Essai sur la politique de la ville, éditions Esprit, 1994) constitue un bon résumé de ces thèses. Sur la structuration des catégories de pensée de la politique de la Ville - et leurs effets - voir la thèse de Sylvie Tissot : Réformer les quartiers. Enquête sociologique sur une catégorie de l'action publique, sous la direction de Christian Topalov, Paris, EHESS, 2002.

44. . Alors que les rodéos des Minguettes n'avaient fait l'objet que d'un entrefilet dans le Monde, le Figaro et l'Humanité (Libération les passe sous silence), la flambée de violence du quartier du Mas du Taureau, à Vaulx-en-Velin, en octobre 1990, donna lieu à 34 reportages et 9 éditoriaux dans la presse audiovisuelle, alors que la presse écrite nationale y consacrait 60 articles. Le volume des articles sur la question du « malaise », $\mathrm{du}$ « mal » ou de « la crise » des banlieues se multiplie dès lors et cela devient un " genre » journalistique à part entière. Sur l'évolution du traitement de ces questions voir Collovald A., « Des désordres sociaux à la violence urbaine ", Actes de la recherche en sciences sociales, $\mathrm{n}^{\circ} 136-137$, mars 2001 . Les médias, notamment audiovisuels, vont 
dès lors jouer un rôle important dans la structuration d'une image publique du problème, en homogénéisant des réalités sociales et géographiques ("les banlieues »); des populations (les « jeunes ») et des faits (les « violences urbaines », les « émeutes ») de statuts très hétérogènes. Voir Champagne $P$., « La construction médiatique des 'malaises sociaux' ", Actes de la recherche en sciences sociales n 90 , décembre 1991, pp. 64-75.

45. . Philippe Juhem montre de la sorte « l'affaiblissement tendanciel de la prééminence des hommes politiques sur les journalistes ", lié à l'alternance politique. Voir Juhem P. SOS-Racisme, histoire d'une mobilisation « apolitique ». Contribution à une analyse des transformations des représentations politiques après 1981, Thèse de science politique sous la direction de Bernard Lacroix, Paris X-Nanterre, 1998.

46. . Rapport sur La politique de la ville, présenté par Gérard Larché, Sénat, session 1992-1993, p. 12.

47. . Le Parisien, 31 octobre 2001.

48. . Interview télévisée de monsieur Jacques Chirac, président de la République à l'occasion de la fête nationale, samedi 14 juillet 2001.

49. . Voir notamment Roché S., Tolérance Zéro ? Incivilités et insécurité, Paris, Odile Jacob, 2002 ; « La théorie de la vitre cassée en France. Incivilités et désordres en public », Revue française de science politique, vol. 50, n³, juin 2000, pp. 387-412. 50. . Wilson J.Q et Kelling G., «Broken Windows : The Police and Neighbourhood Safety ", The Atlantic Monthly, march 1982. Pour une traduction française, voir les Cahiers de la sécurité intérieure, ${ }^{\circ} 15$, 1er trimestre 1994.

51. . Voir par exemple Bousquet R., Insécurité : nouveaux risques. Les quartiers de tous les dangers, Paris, L'Harmattan, 1998 ou Bauer A. et Raufer X., Violences et insécurité urbaine, Paris, PUF (Coll. Que sais-je n 3421), 1998.

52. . Les travaux fins sur les trajectoires de jeunes délinquants - corroborés par des témoignages policiers et judiciaires - font apparaitre dans la majorité des cas une baisse voire une disparition de l'activité délictuelle dès que ces adolescents trouvent un emploi, fondent un couple, etc. Voir par exemple Mauger G., « Espace des styles de vie déviants des jeunes de milieux populaires », in Baudelot C. et Mauger G. (dir.), Jeunesses populaires. Générations de la crise, Paris, L'Harmattan, 1994. Dans un registre différent, on lira avec intérêt Harcourt B. E., " Reflecting on the Subject : A Critique of the Social Influence Conception of Deterrence, the Broken Windows Theory, and OrderMaintenance Policing New-York Style », Michigan Law Review, 97-2, November 1998, pp. 291-389.

53. . Voir Mucchielli L., Violences et insécurité. Fantasmes et réalités dans le débat français, Paris, La découverte, 2001 et Rimbert P., « Les managers de l'insécurité. Production et circulation d'un discours sécuritaire ", in Bonelli L. et Sainati G. (dir.), La machine à punir (...), op. cit.

54. . Salvatore Palidda parle ainsi de « gestion négociée des règles du désordre ». Voir Palidda S., Polizia postmoderna. Etnografia del nuovo controllo sociale, Milan, Feltrinelli, 2000.

55. . La montée en puissance des catégories de la sécurité publique va avoir des effets sur les autres services qui s'occupent du renseignement ou du judiciaire. Sur ce processus, voir notamment Bonelli L., « Les Renseignements généraux et les violences urbaines ", Actes de la Recherche en Sciences Sociales, n¹36-137, mars 2001, pp. 95-103. Pour les tensions inhérentes à la structuration d'un champ des professionnels de la sécurité, voir Bigo D. « La mondialisation de la sécurité ? Réflexions sur le champ 
des professionnels de la gestion des inquiétudes à l'échelle transatlantique et sur ses implications ", TRACES, à paraître, et Bigo D. Polices en réseaux. L'expérience européenne, Paris, Presse de Science-Po, 1996.

56. . Muir W.K., Police : Street Corner Politicians, Chicago, University of Chicago, 1977. 57. . Ces délits consistent respectivement en des insultes et des violences exercées sur des dépositaires de l'autorité. Ils passent sur la période de $0,64 \%$ à $1,08 \%$ de l'ensemble des faits constatés. (Source : Aspects de la criminalité et de la délinquance constatée en France, Paris, La documentation française). Ce calcul reste toutefois trop global, car il agrège des réalités très différentes, notamment entre petites et grandes villes. Sur l'un de mes terrains en banlieue parisienne, l'augmentation est de 470\% entre 1993 et 2001. 58. . La loi pour la sécurité intérieure du 18 mars 2003 (NOR : INTX0200145L) stipule à l'article L. 126-3 que « les voies de fait (...) ou l'entrave apportée, de manière délibérée, à l'accès et à la libre circulation des personnes ou au bon fonctionnement des dispositifs de sécurité et de sûreté, lorsqu'elles sont commises en réunion de plusieurs auteurs ou complices, dans les entrées, cages d'escaliers ou autres parties communes d'immeubles collectifs d'habitation, sont punies de deux mois d'emprisonnement et de 3750 Euros d'amende ». Pour la première fois, le 8 juillet 2003, trois adolescent(e)s de la cité de Brugnauts à Bagneux ont été mis(es) en examen pour « occupation d'une cage d'escalier ». Le 25 juillet, le tribunal correctionnel de Lille condamnait à son tour deux jeunes de 19 ans à un mois de prison ferme pour « occupation illicite des parties communes d'un immeuble ».

59. . Le traitement en temps réel, DACG, ministère de la Justice, p. 3.

60. . Ibid., p. 4.

61. . Mary P., « Pénalité et gestion des risques : vers une justice 'actuarielle' en Europe? », Déviance et société, vol. 25, n¹, 2001, p. 35.

62. . Voir Sainati G., « Des techniques aux pratiques de pénalisation de la pauvreté » in Bonelli L. et Sainati G. (dir), La machine à punir (...), op. cit., pp. 87-105.

63. . Pistolet spécial tirant des balles en caoutchouc.

64. . Insécurité : les nouveaux risques, op. cit., pp. 121-122. Il est repris en cela par des journalistes à sensation, comme Christian Jelen, par exemple, qui n'hésite pas à intituler son ouvrage La guerre des rues (Paris, Plon, 1999).

65. . Le rapport 2002 du Comité pour les droits, la justice et les libertés de Saint Denis (Seine Saint Denis) donne de nombreux témoignages de ces relations mimétiques tant au niveau du langage (" pourquoi tu m'as regardé ? ; « alors, c'est qui le chef ?»; « viens te battre si t'es un homme ») que des pratiques. On trouvera d'autres exemples dans le rapport de la commission d'enquête sur les comportements policiers à Châtenay-Malabry, Poissy et Paris 20e (juillet 2002), menée par la Ligue des droits de l'homme, le syndicat des avocats de France et le syndicat de la magistrature. 66. . Voir Jobard F., Bavures policières ? La force publique et ses usages, Paris, La découverte, 2002.

67. Contrat local de sécurité de la ville de Chalon sur Saône - Document de travail page 66 - 26 septembre 2002 - Annexe 4 : Note sur le suivi personnalisé des familles et mineurs signalés.

68. . Goffman E., Asiles. Etudes sur la condition sociale des malades mentaux, Paris, éditions de Minuit, 1968, pp. 214-218.

69. . Pierre Bourdieu rappelle de la sorte qu'on ne peut pas rendre compte des dispositions et des pratiques des adolescents des quartiers populaires, et notamment des plus « déviantes », sans faire intervenir d'autres facteurs, au premier rang desquels 
« le dépérissement ou l'affaiblissement des instances de mobilisation, telles les organisations politiques et syndicales qui, dans les anciennes 'banlieues rouges', ne se contentaient pas seulement, comme on le dit souvent de 'canaliser et de réguler la révolte', mais assuraient une sorte 'd'enveloppement continu' de toute l'existence (à travers notamment l'organisation des activités sportives, culturelles et sociales), contribuant ainsi à donner un sens à la révolte, mais aussi à toute l'existence ». Bourdieu P. (dir.), La misère du monde, Paris, Seuil, 1993, p. 225.

70. . Edelman M., Pièces et règles du jeu politique, Paris, Seuil, 1991, pp. 53 et suiv. 71. . « there is no limit to police participation in the construction and management of social problems. [The police are] shaping the knowledges requirements of other institutions in order to assist those institutions in the risk management of the special populations for whom they are responsible ». Ericson R. V. and Haggerty K. D., Policing the Risk Society, University of Toronto Press, 1997, pp. 73 et 75.

72. . Castel R., Les métamorphoses de la question sociale. Une chronique du salariat, op. cit. p. 163.

73. . Christie N., L'industrie de la punition. Prison et politique pénale en Occident, Paris, Autrement, 2003.

74. . Ce qui diffère fondamentalement des groupes organisés, qu'ils soient « criminels » ou « terroristes ». En cas d'arrestations, reconstituer un groupe dont la cohérence repose sur des relations de confiance à la hauteur des risques encourus est en effet beaucoup plus long et compliqué.

75. . Pour une présentation de ces thèses, voir Wacquant L., « Les mythes savants du nouveau sécuritarisme », Les politiques sociales, $\mathrm{n}^{\circ} 1 \& 2-2003$.

76. . En 2002, l'inspection générale de la police nationale (IGPN) a enregistré 592 plaintes pour violences policières illégitimes, contre 566 en 2001 et 548 en 2000, soit une hausse de $8 \%$ en trois ans. Cette évolution est encore plus flagrante pour l'inspection générale des services (IGS) qui s'occupe de Paris et de la petite couronne : 432 dossiers en 2001, 385 en 2001, 360 en 2000, et 216 en 1997. En cinq ans, ce chiffre a donc été multiplié par deux. (Source, Le Monde, 21 février 2003).

77. . Voir Bigo D., « Sécurité et immigration. Vers une gouvernementalité par l'inquiétude ? ", Cultures \& Conflits n³2-32, automne 1998 et Delumeau J., Rassurer et protéger : le sentiment de sécurité dans l'Occident d'autrefois, Paris, Fayard, 1989.

78. . Beaud S. et Pialoux M. Violences urbaines, violences sociales. Genèse des nouvelles classes dangereuses, Paris, Fayard, 2003.

\section{INDEX}

Index géographique : France

Mots-clés : criminalité, délinquance, Etat providence

Index chronologique : 1980 - 1990, 1990 - 2000 